\section{Lymphknotenmetastasen bei Prostatakarzinom - zwei neue radiologische Methoden im Vergleich}

\author{
Sowohl die Kombination aus ${ }^{11} \mathrm{C}-\mathrm{C}$-Chin-Positronenemissionstomografie und \\ Computertomografie $\left({ }^{11} \mathrm{C}-\mathrm{Cholin}-\mathrm{PET} / \mathrm{CT}\right)$ als auch die Ferumoxtran-10-ver- \\ stärkte Magnetresonanz-Lymphografie werden zur lymphknotendiagnostik \\ eingesetzt. Was leisten sie?
}

\begin{abstract}
n der niederländisch-deutschen Studie wurden 29 Prostatakarzinompatienten untrsucht: 22 mit einem Rezidiv, sieben mit einem Ersttumor. Zur Detektion befallener Lymphknoten erfolgte eine Diagnostik mit zwei neuen Verfahren: Bei der Ferumoxtran-10-verstärkten Magnetresonanz-Lymphografie (MRL) wird Ferumoxtran-10 von Makrophagen aufgenommen und nur in nicht befallene Lymphknoten transportiert. Die ${ }^{11} \mathrm{C}$ Cholin-PET/CT beruht dagegen auf der spezifischen Einlagerung von ${ }^{11} \mathrm{C}$-Cholin in Tumorzellen. Die Befunde der PET/CT beurteilten Nuklearmediziner, die der
\end{abstract}

MRL Uro-Radiologen. Als Referenzkriterium für die Malignität eines Lymphknotens stand kein histologischer Befund zur Verfügung. Stattdessen prüfte ein unabhängiger Radiologe alle in der Signalgebung verdächtigen Befunde und legte bei ihnen einen Cut-off-Wert von $\geq 7 \mathrm{~mm}$ für den Lymphknotendurchmesser als positives Malignitätskriterium fest.

Durch die MRL wurden 738 Lymphknoten erkannt und 151 Knoten - bei 23 der Patienten (79\%) - als positiv gewertet. Die PET/CT führte bei 132 sichtbaren Lymphknoten zu 34 positiven Befunden - bei 13 der Studienteilnehmer
(45\%). Diese Unterschiede waren ebenso signifikant wie der kleinere Durchmesser verdächtiger Lymphknoten von im Mittel 4,9 mm in der MRL gegenüber 8,4 $\mathrm{mm}$ bei der PET/CT.

$61 \%$ der Patienten hatten auffällige MRL-Befunde, die außerhalb des üblichen Zielvolumens für die Strahlentherapie des Beckens lagen. Bei PET/CT traf dies auf $31 \%$ der Patienten zu.

Fazit: Limitierend an dieser Studie ist, dass als Referenzkriterium für Malignität keine histologische Beurteilung der Lymphknoten stattfand. Die MRL scheint trotzdem der PET/CT überlegen $\mathrm{zu}$ sein. Auffällig ist der große Anteil verdächtiger Lymphknoten außerhalb des radiotherapeutischen Zielvolumens.

Andreas Fischer

Fortuin AS et al. Value of PET/CT and MR lymphography in treatment of prostate cancer patients with lymph node metastases. Int J Radiat Oncol Biol Phys. 2012;84(3):712-8.

\title{
Lebensqualität steigt im Zeitverlauf wieder an
}

\section{Die Diagnose Prostatakarzinom beeinträchtigt bekanntermaßen die Lebensqualität älterer Männer. In einer Studie wurden erstmals Daten ausgewertet, die bereits vor der Diagnose erfasst wurden sowie Veränderungen über einen längeren Zeitraum.}

G rundlage der Analyse waren Daten von 445 Patienten, die bereits vor der Diagnose Prostatakarzinom am USamerikanischen Medicare Health Outcomes Survey (MHOS) teilgenommen hatten und den Short Form-36-Fragebogen (SF-36) zur Lebensqualität zum ersten Mal schon vor der Tumordiagnose beantwortet hatten. Als Kontrollen dienten für jeden Patienten mit Prostatakarzinom jeweils fünf MHOS-Teilnehmer ohne Tumorerkrankung mit sonst vergleichbaren medizinischen und psychosozialen Charakteristika.

Im Vergleich zur Erstuntersuchung hatte bei den noch lebenden Patienten die physische Lebensqualität innerhalb der ersten sechs Monate nach Diagnose abgenommen, v. a. in den Bereichen körperliche Rollenfunktion (-7,6 vs. $-3,0$ bei den Kontrollen), allgemeine Gesundheit $(-5,0$ vs. $-1,7)$ und physischer Gesamt- score $(-4,9$ vs. 2,1$)$. Die mentale Lebensqualität sank sechs bis zwölf Monate nach der Diagnose am deutlichsten. Dabei waren vor allem die soziale Kompetenz ( $-4,8$ vs. $-1,4$ bei den Kontrollen), die Vitalität $(-3,7$ vs. $-1,4)$ und die mentale Gesamtkomponente $(-3,7$ vs. 1,1$)$ betroffen.

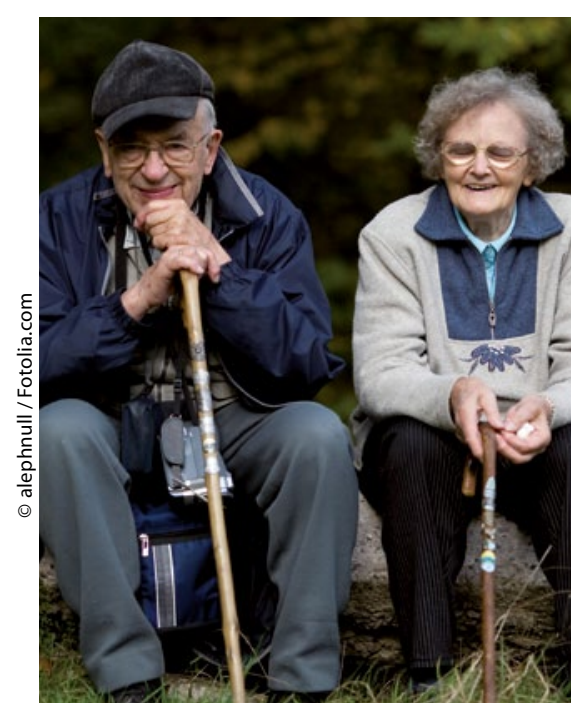

Physische und psychische Lebensqualität nahmen nach zwölf Monaten wieder $\mathrm{zu}$ und erreichten nach eineinhalb Jahren mit den Kontrollprobanden vergleichbare Werte. Nach dieser Zeit hatte allerdings das Risiko für eine Depression zugenommen. Es war gegenüber der Erstuntersuchung v. a. nach externer Strahlentherapie und bei konservativem Prozedere um mehr als das Dreifache erhöht.

Fazit: Bei älteren Prostatakarzinompatienten nimmt im Vergleich zu Kontrollen die Lebensqualität im ersten Jahr ab. Nach eineinhalb Jahren nimmt sie bei den Überlebenden jedoch wieder deutlich zu und erreicht fast das Niveau der Vergleichsgruppe.

Andreas Fischer

Reeve BB et al. Impact of diagnosis and treatment on clinically localized prostate cancer on health-related quality of life for older Americans. Cancer. 2012;118(22):5679-87.

Die Lebensqualität älterer Männer nimmt durch die Diagnose Prostatakarzinom zunächst ab - später kann sie aber wieder zunehmen. 